\section{(6) OPEN ACCESS}

\title{
Genome-wide DNA methylation analysis in multiple tissues in primary Sjögren's syndrome reveals regulatory effects at interferon-induced genes
}

\author{
Juliana Imgenberg-Kreuz, ${ }^{1}$ Johanna K Sandling, ${ }^{1,2}$ Jonas Carlsson Almlöf, ${ }^{1}$ \\ Jessica Nordlund ${ }^{1}{ }^{1}$ Linnea Signér ${ }^{2}$ Katrine Braekke Norheim, ${ }^{3}$ Roald Omdal, ${ }^{3}$ \\ Lars Rönnblom, ${ }^{2}$ Maija-Leena Eloranta, ${ }^{2}$ Ann-Christine Syvänen, ${ }^{1}$ Gunnel Nordmark ${ }^{2}$
}

\begin{abstract}
Handling editor Tore K Kvien
- Additional material is published online only. To view please visit the journal online (http://dx.doi.org/10.1136/ annrheumdis-2015-208659).
\end{abstract}

${ }^{1}$ Molecular Medicine and Science for Life Laboratory, Department of Medical Sciences, Uppsala University, Uppsala, Sweden

${ }^{2}$ Rheumatology and Science for Life Laboratory, Department of Medical Sciences, Uppsala University, Uppsala, Sweden ${ }^{3}$ Clinical Immunology Unit, Department of Internal Medicine, Stavanger University Hospital, Stavanger, Norway

\section{Correspondence to} Dr Gunnel Nordmark

Rheumatology and Science for Life Laboratory, Department of Medical Sciences, Uppsala University, Uppsala SE-751 85 Sweden;

Gunnel.Nordmark@medsci.uu.

$J-K$ and JKS contributed equally.

Received 25 September 2015 Revised 11 January 2016 Accepted 16 January 2016 Published Online First 8 February 2016

\section{ABSTRACT}

Objectives Increasing evidence suggests an epigenetic contribution to the pathogenesis of autoimmune diseases, including primary Sjögren's Syndrome (pSS). The aim of this study was to investigate the role of DNA methylation in pSS by analysing multiple tissues from patients and controls.

Methods Genome-wide DNA methylation profiles were generated using HumanMethylation450K BeadChips for whole blood, CD19+ B cells and minor salivary gland biopsies. Gene expression was analysed in CD19+ B cells by RNA-sequencing. Analysis of genetic regulatory effects on DNA methylation at known pSS risk loci was performed.

Results We identified prominent hypomethylation of interferon (IFN)-regulated genes in whole blood and CD19+ B cells, including at the genes MX1, IFI44L and $P A R P 9$, replicating previous reports in $\mathrm{pSS}$, as well as identifying a large number of novel associations.

Enrichment for genomic overlap with histone marks for enhancer and promoter regions was observed. We showed for the first time that hypomethylation of IFNregulated genes in pSS B cells was associated with their increased expression. In minor salivary gland biopsies we observed hypomethylation of the IFN-induced gene OAS2 Pathway and disease analysis resulted in enrichment of antigen presentation, IFN signalling and

lymphoproliferative disorders. Evidence for genetic control of methylation levels at known pSS risk loci was observed. Conclusions Our study highlights the role of epigenetic regulation of IFN-induced genes in pSS where replication is needed for novel findings. The association with altered gene expression suggests a functional mechanism for differentially methylated CpG sites in pSS aetiology.

\section{INTRODUCTION}

Primary Sjögren's syndrome (pSS) is a chronic autoimmune disease characterised by inflammation of salivary and lacrimal glands. Systemic manifestations such as arthritis, pulmonary or renal involvement may also occur. ${ }^{1}$ The aetiology of pSS is multifactorial, where both genetic and environmental factors are thought to contribute to disease development. However, the molecular mechanisms underlying pSS remain largely elusive. ${ }^{2}$ Activation of the type I interferon (IFN) system with elevated expression of type I IFN-regulated genes in blood cells and minor salivary glands, a so-called IFN signature, has been demonstrated in $\mathrm{pSS}^{3-7} \mathrm{~B}$ cell activation in pSS is reflected by autoantibody synthesis and an increased risk of non-Hodgkin's lymphoma, most commonly of the B cell type. ${ }^{8}$

For pSS, to date, about 10 genetic risk loci have been identified through genome-wide association studies (GWAS). ${ }^{9} 10$ These variants only explain a limited proportion of the susceptibility to pSS, and the functional consequence of associated single nucleotide polymorphisms (SNPs) remains unclear for most loci. ${ }^{11}$ Increasing evidence suggests an epigenetic contribution to the pathogenesis of autoimmune diseases, including pSS. ${ }^{12}$ Epigenetic modifications constitute an additional layer of genomic regulation, and may serve as a dynamic link between genotype, environment and phenotype, for example by modulating gene expression. Methylation of the DNA base cytosine $(5 \mathrm{mC})$ can be studied in large sample sets with array-based methods such as the Illumina HumanMethylation450K array (HM450K), which allows for quantification of DNA methylation of $485577 \mathrm{CpG}$ sites across the human genome. ${ }^{13}$

In pSS, so far, two studies with relatively small sample sizes have applied the HM450K array. ${ }^{14} 15$ Altorok et $a l^{14}$ report a number of differentially methylated $\mathrm{CpG}$ sites (DMCs) in naïve CD4+ T cells from 11 patients and 11 controls, with implications in immune responses and lymphocyte activation, including hypomethylation of IFNregulated genes. Miceli-Richard et $a l^{15}$ found more prominent methylation changes in CD19+ B cells than in CD4 + T cells in 26 patients compared with 22 controls.

In order to evaluate the role of DNA methylation in pSS in a comprehensive manner we performed an epigenome-wide association study (EWAS) in whole blood, CD19+ B-cells and minor salivary gland biopsy samples from patients and controls using the HM450K array. To further explore the functional role of the DMCs we intersected the diseaseassociated $\mathrm{CpG}$ sites with publicly available chromatin state data ${ }^{16}$ and performed gene expression analysis in CD19+ B cells. Finally, genetic regulation of methylation at pSS risk loci was investigated.

\section{PATIENTS AND METHODS}

For full details of methods see online supplementary text. 


\section{Patients and controls}

A total of 108 Caucasian patients with pSS from the rheumatology clinic at the Uppsala University Hospital, Sweden, were included in the study, all fulfilling the American European Consensus Group (AECG) criteria. ${ }^{17}$ Whole blood was collected from 100 patients, CD19+ B cells from 24 patients and minor salivary gland biopsies from 15 patients. As control samples, DNA from whole blood from 400 healthy blood donors from the Uppsala Bioresource and CD19+ B cells from 47 donors were analysed. ${ }^{18}$ Only controls falling within the main European cluster in our previous study were included..$^{19}$ Minor salivary gland biopsies obtained from 13 individuals that were examined for a possible pSS diagnosis, where the biopsies showed no inflammation and serology was negative for autoantibodies, served as control biopsies (table 1).

\section{Genome-wide methylation analysis}

Genomic DNA from whole blood, CD19+ B cells and minor salivary gland biopsies was isolated using standard procedures. DNA methylation levels of $485577 \mathrm{CpG}$ sites were determined on the HM450K BeadChip (Illumina, San Diego, California, USA). Signal intensities were parsed into the Minfi $\mathrm{R}$ package for Subset-quantile Within Array Normalization. ${ }^{20-22}$ The postquality control (QC) data set comprised $388971 \mathrm{CpG}$ sites. To determine differential methylation between patients with pSS and controls a linear regression model containing cell count estimates, ${ }^{23}{ }^{24}$ age and sex as covariates was fitted. DMCs with a Bonferroni-adjusted threshold of $\mathrm{p}<1.3 \times 10^{-7}$ were considered significant.

\section{Gene expression profiling of CD19+ B cells}

Expression analysis on CD19+ B cells $(n=16$ patients, $n=23$ controls) was conducted using the TruSeq stranded mRNA sample preparation kit followed by sequencing on a HiSeq2500 instrument (Illumina). QC was conducted using RNA-seQC. ${ }^{25}$ Reads were mapped with Tophat 2 and analysis of differential gene expression was performed using the Cufflinks pipeline. ${ }^{26} 27$

\section{Methylation quantitative trait loci analysis}

Methylation levels were tested in PLINK for genotype association at loci that have previously shown an association with pSS with genome-wide significance. ${ }^{9} 28$ Quality controlled genotype data for 135503 probes generated on the Infinium ImmunoChip (Illumina) were available for 382 of the healthy control individuals in our study. All CpG sites within a gene locus plus $100 \mathrm{~kb}$ flanking regions were tested against all genotypes within the same region. A Bonferroni corrected $\mathrm{p}<1.24 \times 10^{-7}$ was considered statistically significant.

\section{RESULTS}

\section{Differential methylation in whole blood}

First, we investigated the difference in methylation levels in whole blood between patients with pSS and controls. We used reference DNA methylation signatures of flow sorted blood cells types to estimate cell counts and found reduced $\mathrm{CD} 4+$ and CD8 + T cells in pSS, while CD19+ B cell proportions were estimated to be similar between patients and controls (see online supplementary figure S1). We identified 11785 (6171 hypomethylated and 5614 hypermethylated) DMCs annotated to 5623 unique genes (see figure 1 and online supplementary table S1).

An average difference in $\beta$-values of $>0.1$ between cases and controls was identified at 12 of the 11785 DMCs, of which 11
DMCs, annotated to seven different genes, were hypomethylated in pSS (table 2). The most pronounced difference in methylation was detected for a CpG site annotated to $M X$ dynamin-like GTPase 1 (MX1) (also referred to as MxA). MX1 is a key mediator of human antiviral immune responses and is induced by type I and type II IFNs. ${ }^{29}$ Two additional CpG sites in MX1 were found to be distinctly hypomethylated in patients. Of interest, among the top hypomethylated DMCs in pSS we further note $\mathrm{CpG}$ sites in the IFN-induced genes IFI $44 L$, PARP9, PLSCR1, IFIT1, IFITM1 and HLA-A, meaning that all of the top hypomethylated sites in pSS are IFN-regulated (table 2). In addition, we detected a large number of DMCs in IFN-induced genes with a difference in methylation $<0.1$, for example, STAT4, NFAT5, ELF1, OAS1-3 and TREX1. Multiple DMCs with a difference in methylation $<0.1$ were also observed in the human leucocyte antigen (HLA) region, both major histocompatibility complex (MHC) class I and class II, the majority being hypomethylated in the patients (see online supplementary table S1). We identified one hypermethylated CpG site with an average difference in methylation- $\beta$ of $>0.1$ annotated to $E B F 4$, which is a transcription factor belonging to the Olf-1/EBF family with central implications in neural development and B cell maturation (table 2). ${ }^{30}$

We then analysed the 12 top differentially methylated sites in patients with pSS stratified on the presence of anti-Sjögren's Syndrome antigen A (SSA) and/or anti-Sjögren's Syndrome antigen B (SSB)-antibodies. When analysing antibody-positive patients versus controls, a more prominent difference in mean methylation was seen, indicating that the difference in mean methylation for the IFN-induced genes is mainly driven by the antibody-positive patients (see online supplementary table S2). Analysing only patients with pSS $(\mathrm{n}=57)$ with early disease defined as $\leq 3$ years from diagnosis to blood sampling, almost identical results of difference in mean methylation were seen as in the analysis of all 100 cases versus controls (data not shown).

Association analysis of sex-chromosomal CpG sites was conducted separately for female and male individuals. We identified $85 \mathrm{X}$ chromosomal CpG sites (out of $11232 \mathrm{X}$ chromosomal sites included on the HM450K array), annotated to 56 unique genes to be differentially methylated in female patients compared with female controls, with DMCs in notable genes such as VSIG4, TLR8, CD4OL as well as in several microRNAs (miRNAs) (see online supplementary table S3). There were no DMCs in male individuals on the $\mathrm{X}$ chromosome and the $\mathrm{Y}$ chromosome.

Pathway analysis of the 500 most significantly associated DMCs in whole blood identified antigen presentation, IFN signalling and graft-versus-host disease signalling as the top canonical pathways (see online supplementary table S4). The strongest gene-set enrichment in disease or function annotation of DMCs was observed for lymphohaematopoietic cancer $\left(p=6 \times 10^{-13}\right)$ (see online supplementary table S5). Given the large number of DMCs between patients and controls, we analysed global DNA methylation levels in whole blood, CD19+ B cells and minor salivary gland biopsies and found no difference between patients and controls (see online supplementary figure S2).

\section{Functional genomic distribution and overlap with chromatin marks}

In general, DMCs were enriched in $\mathrm{CpG}$ island shelves and open sea regions and depleted in $\mathrm{CpG}$ islands and shores (figure 2A). Investigating the distribution of hypomethylated and hypermethylated DMCs separately, hypomethylated DMCs were over-represented in 5'-untranslated region (UTR), whereas 
Table 1 Characteristics of patients with primary Sjögren's syndrome (pSS) and control individuals

\begin{tabular}{|c|c|c|c|c|c|c|}
\hline & \multicolumn{2}{|l|}{ Whole blood } & \multicolumn{2}{|l|}{ CD19+ B cells } & \multicolumn{2}{|c|}{ Minor salivary gland biopsies } \\
\hline & Patients with pSS & Controls & Patients with pSS & Controls & Patients with pSS & Controls \\
\hline Individuals, n & 100 & 400 & 24 & 47 & 15 & 13 \\
\hline Women, n (\%) & $89(89)$ & $351(87.8)$ & $24(100)$ & $35(74.4)^{*}$ & $15(100)$ & $13(100)$ \\
\hline Age, years, $($ mean $\pm S D)$ & $56.1 \pm 13.6$ & $47.1 \pm 13.2^{* *}$ & $56.6 \pm 13.8$ & $48.8 \pm 17.7$ & $47.8 \pm 15.9$ & $47.5 \pm 17.2$ \\
\hline \multicolumn{7}{|l|}{ Autoantibody frequency, n (\%) } \\
\hline Antinuclear antibodies (ANAs) & $80(80)$ & n.a. & $20(83.3)$ & n.a. & $14(93.3)$ & 0 \\
\hline Anti-SSA antibodies & $75(75)$ & n.a. & $24(100)$ & n.a. & $15(100)$ & 0 \\
\hline Anti-SSB antibodies & $41(41)$ & n.a. & $13(54.2)$ & n.a. & $8(53.3)$ & 0 \\
\hline \multicolumn{7}{|l|}{ Minor salivary gland biopsiest } \\
\hline Focus score, median (range) & $2(1-12)$ & n.a. & $2(1-12)$ & n.a. & $2(1-10)$ & 0 \\
\hline \multicolumn{7}{|l|}{ Medication, n (\%) } \\
\hline Prednisolone & $7(7)$ & n.a. & $1(4.2)$ & n.a. & 0 & n.a. \\
\hline Hydroxychloroquine & $14(14)$ & n.a. & $5(20.8)$ & n.a. & $1(6.7)$ & n.a. \\
\hline Immunosuppressants & $4(4) \ddagger$ & n.a. & $2(8.4) \S$ & n.a. & 0 & n.a. \\
\hline
\end{tabular}

Whole blood and CD19+ B cells from the same individual were available from 17 patients; whole blood, CD19+ B cells and biopsies from 10 patients.

${ }^{*} p<0.01,{ }^{*} p<0.0001$ for the difference between patients with pSS and controls. Frequencies compared with Fisher's exact test and continuous variables with Mann-Whitney $U$ test.

tBiopsies performed in 75/100 patients with whole blood samples and 21/24 patients with CD19+ B cells.

$\ddagger$ Azathioprine $(n=2)$, mycophenolate mofetil $(n=1)$, chlorambucil $(n=1)$.

§Methotrexate $(n=2)$.

n.a., not available.

hypermethylated DMCs were more than twofold enriched in $3^{\prime}$-UTR and moderately enriched in gene bodies (figure 2B). Analysing the intersection of pSS associated CpG sites with chromatin marks revealed that DMCs with hypomethylation in patients were enriched in enhancers (H3K4me1 and H3K27ac) and accessible chromatin (DNase I hypersensitive sites, DHS) compared with the distribution of all probes on the array. In contrast, hypermethylated DMCs were depleted for these modifications and also largely under-represented in the active promoter mark H3K4me3. On the other hand, DMCs with hypermethylation in patients were enriched for $\mathrm{H} 3 \mathrm{~K} 36 \mathrm{me} 3$, which marks an actively transcribed gene body (figure 2C).

\section{Differential methylation and mRNA expression in CD19+ \\ B cells}

Next, we analysed primary CD19+ B cells from 24 patients and 47 healthy controls and found 453 DMCs, $(98$ hypomethylated and 355 hypermethylated, annotated to 303 unique genes) (see online supplementary table S6). The top associated DMCs are shown in table 3. Similar to whole blood, several IFN-induced genes showed prominent hypomethylation at multiple $\mathrm{CpG}$ sites in CD19+ B cells from patients with pSS. In order to investigate whether differential methylation was associated with gene expression, gene expression analysis was performed in CD19+ $B$ cells from a subset of patients and controls. Significantly upregulated expression was observed for all of the eight IFN-induced genes exhibiting DMCs with hypomethylation $>0.2$ (table 3 ). In contrast, for the two genes with hypermethylated DMCs $>0.2$, no significant association with gene expression was observed.

\section{Differential methylation in minor salivary gland biopsies}

Finally, we studied DNA methylation directly in the primary target organ of the disease, the salivary gland. Given the

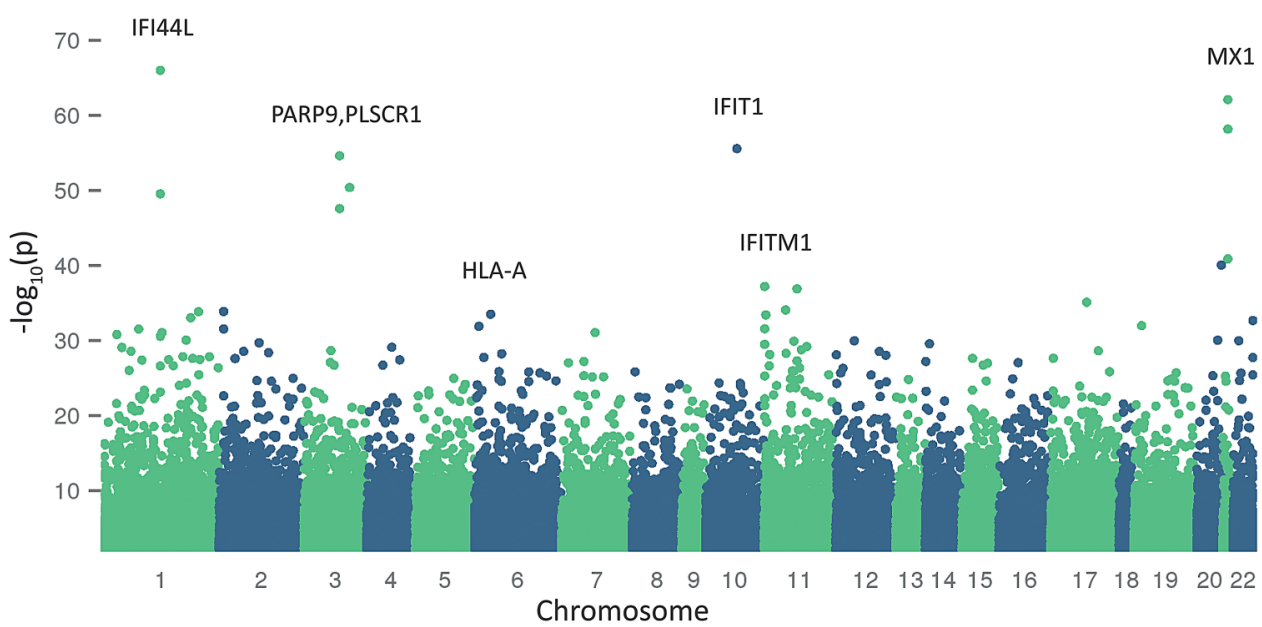

Figure 1 Manhattan plot of the genome-wide DNA methylation analysis in whole blood from patients with primary Sjögren's syndrome (pSS) and control individuals. The figure shows the $-\log _{10} p$ values of the association of CpG sites with pSS against the chromosomal position of the investigated sites. The hypomethylated CpG sites with a difference in mean methylation- $\beta>0.1$ are highlighted by their gene symbol annotation. 
Table 2 Differentially methylated $\mathrm{CpG}$ sites (DMCs) with the largest difference in mean methylation values between patients with primary Sjögren's syndrome (pSS) and control individuals in whole blood $(\Delta \beta$-value $>0.1)$

\begin{tabular}{|c|c|c|c|c|c|c|c|c|c|}
\hline CpG site & $\begin{array}{l}\text { Gene } \\
\text { symbol }\end{array}$ & Gene name & Interferon-induced & $\begin{array}{l}\text { CpG island } \\
\text { information }\end{array}$ & $\begin{array}{l}\text { Gene } \\
\text { property }\end{array}$ & $\begin{array}{l}\text { Mean } \\
\text { methylation } \beta \\
\text { in pSS }\end{array}$ & $\begin{array}{l}\text { Mean } \\
\text { methylation } \beta \\
\text { in controls }\end{array}$ & $\begin{array}{l}\text { Methylation } \\
\Delta \boldsymbol{\beta}^{*}\end{array}$ & $p$ Value \\
\hline $\operatorname{cg} 21549285$ & $M X 1$ & $\begin{array}{l}\text { MX dynamin-like } \\
\text { GTPase } 1\end{array}$ & Yes & Shore & $5^{\prime}$ UTR & 0.57 & 0.83 & -0.26 & $6.9 \times 10^{-59}$ \\
\hline cg05696877 & IFI44L & $\begin{array}{l}\text { Interferon-induced } \\
\text { protein } 44 \text {-like }\end{array}$ & Yes & Open sea & $5^{\prime}$ UTR & 0.47 & 0.67 & -0.20 & $2.8 \times 10^{-50}$ \\
\hline $\operatorname{cg} 22930808$ & PARPg & $\begin{array}{l}\text { Poly (ADP-ribose) } \\
\text { polymerase family, } \\
\text { member } 9\end{array}$ & Yes & Shore & $5^{\prime}$ UTR & 0.52 & 0.71 & -0.19 & $2.4 \times 10^{-55}$ \\
\hline $\operatorname{cg} 22862003$ & $M X 1$ & $\begin{array}{l}\text { MX dynamin-like } \\
\text { GTPase } 1\end{array}$ & Yes & Shore & TSS1500 & 0.51 & 0.70 & -0.19 & $7.9 \times 10^{-63}$ \\
\hline cg03607951 & IFI44L & $\begin{array}{l}\text { Interferon-induced } \\
\text { protein } 44 \text {-like }\end{array}$ & Yes & Open sea & TSS1500 & 0.41 & 0.59 & -0.18 & $9.9 \times 10^{-67}$ \\
\hline cg00959259 & PARP9 & $\begin{array}{l}\text { Poly (ADP-ribose) } \\
\text { polymerase family, } \\
\text { member } 9\end{array}$ & Yes & Shore & $5^{\prime}$ UTR & 0.41 & 0.58 & -0.17 & $2.6 \times 10^{-48}$ \\
\hline $\operatorname{cg} 26312951$ & $M X 1$ & $\begin{array}{l}\text { MX dynamin-like } \\
\text { GTPase } 1\end{array}$ & Yes & Shore & TSS200 & 0.29 & 0.44 & -0.14 & $1.3 \times 10^{-41}$ \\
\hline $\operatorname{cg} 23570810$ & IFITM1 & $\begin{array}{l}\text { Interferon-induced } \\
\text { transmembrane protein } \\
1\end{array}$ & Yes & Shore & $\begin{array}{l}\text { Gene } \\
\text { body }\end{array}$ & 0.55 & 0.69 & -0.14 & $6.1 \times 10^{-38}$ \\
\hline $\operatorname{cg} 17608381$ & $H L A-A$ & $\begin{array}{l}\text { Major histocompatibility } \\
\text { complex, class I, A }\end{array}$ & Yes & Shore & $\begin{array}{l}\text { Gene } \\
\text { body }\end{array}$ & 0.48 & 0.60 & -0.12 & $3.4 \times 10^{-15}$ \\
\hline cg05825244 & EBF4 & Early B cell factor 4 & No & CpG island & $\begin{array}{l}\text { Gene } \\
\text { body }\end{array}$ & 0.57 & 0.47 & 0.11 & $2.4 \times 10^{-11}$ \\
\hline
\end{tabular}

${ }^{*}$ Methylation $\Delta \beta$ refers to the difference in mean methylation $\beta$ ( $\beta$-values) between patients with pSS and healthy controls. A negative $\Delta \beta$ represents hypomethylation in the patients. TSS, transcription start site.

described IFN signature in the minor salivary glands, we hypothesised there might be epigenetic changes in IFN-induced genes. $^{45}$ We found 45 DMCs annotated to 19 unique genes, where the most significant DMC showed hypomethylation in OAS2, which encodes a member of the IFN-inducible $2^{\prime}-5^{\prime} \mathrm{A}$ synthetase family, involved in the innate immune response to viral infections (see online supplementary table S7). ${ }^{31}$

\section{Genetic regulation of DNA methylation at pSS risk loci}

To investigate whether the effects of established pSS risk alleles are mediated through changes in DNA methylation, we analysed genetic variants in 382 of our control individuals at the following pSS GWAS loci: DDX6-CXCR5, FAM167A-BLK, IL12A, IRF5-TNPO3, STAT4, TNIP1, and within the HLA region, for association with methylation levels in whole blood. Evidence for genetic regulation of DNA methylation, that is, significant methylation quantitative trait loci (metQTL), was identified for all pSS risk loci (in total 36679 metQTL). Table 4 shows the metQTL for the pSS associated SNPs. ${ }^{9}$ Apart from the HLA region, the most significant metQTL was observed for the IRF5-TNPO3 SNP rs4728142 and CpG site cg04864179, both located in the IRF5 promoter. Interestingly, the IRF5-TNPO3 locus is also the non-HLA gene locus most significantly associated with pSS. ${ }^{9}$ Methylation levels in blood at cg04864179 were also directly associated with pSS in our EWAS $\left(p=4.8 \times 10^{-13}\right.$, mean methylation- $\beta$ difference 0.04$)$.

\section{DISCUSSION}

Here we report a comprehensive analysis of DNA methylation in pSS; in whole blood, primary CD19+ B cells and minor salivary gland biopsies. The most prominent finding was hypomethylation of IFN-regulated genes, including multiple associated $\mathrm{CpG}$ sites in MX1 and IFI44L, replicating previous reports in pSS. ${ }^{14} 15$ Notably, all top DMCs were annotated to IFN-induced genes. We also identified numerous significant, but smaller methylation differences between patients and controls most of which are novel (methylation- $\beta$ value differences $<0.1$, online supplementary table S1). We show for the first time that hypomethylation of IFN-regulated genes in pSS CD19+ B cells is correlated with increased gene expression. PSS belongs to the systemic autoimmune diseases, together with systemic lupus erythematosus (SLE) which display an IFN signature, with several possible mechanisms underlying the IFN activation. ${ }^{32-34}$ Recently, MX1 has been suggested as a potential biomarker for disease activity and type I IFN bioactivity in pSS, ${ }^{35}$ and IFI44L is described as an indicator gene of the type I IFN signature.? Hypomethylation in IFI44L, which was initially reported by Altorok et $a l^{14}$ in pSS CD4+ T cells and subsequently by Miceli-Richard et $a l^{15}$ in CD19+ B cells, has also been detected in multiple cell types from patients with SLE. ${ }^{36-39}$ Absher $e t a l^{37}$ found hypomethylation of IFN-induced genes in naïve, memory and regulatory CD4 + T cells, CD19+ B cells and CD14+ monocytes from patients with SLE, with either active or quiescent disease. They conclude that epigenetic changes occur in 
Figure 2 Functional genomic distribution of differentially methylated CpG sites (DMCs) in whole blood from patients with primary Sjögren's syndrome (pSS) and controls. This figure illustrates the functional genomic distribution of all associated autosomal DMCs (first panel), of the hypomethylated DMCs (second panel) and of the hypermethylated DMCs (third panel) annotated (A) in relation to $\mathrm{CpG}$ island context, $(\mathrm{B})$ in relation to gene region and $(C)$ in relation to chromatin states in CD19+ B and CD3+ T reference cells. The colour scale indicates fold-enrichment (orange) or fold-depletion (blue) of the DMCs in each functionally annotated region. The bold numbers represent annotations to which the DMCs significantly differ compared with the distribution of probes on the HM450K array (post-QC probe set) (Bonferroni corrected $X^{2}$-test $p$ value $<0.005$ ). TSS1500, 1500 bp upstream of transcription start site (TSS); TSS200, 200 bp upstream of TSS; ncRNA, non-coding RNA; NA, probe not annotated to a defined gene property.
A

\begin{tabular}{c|c|c|}
$\begin{array}{c}\text { All associated } \\
\text { DMCs }\end{array}$ & \multicolumn{1}{c}{$\begin{array}{c}\text { Hypometh. } \\
\text { DMCs }\end{array}$} & $\begin{array}{c}\text { Hypermeth. } \\
\text { DMCs }\end{array}$ \\
\hline 0.29 & 0.49 & 0.08 \\
\hline 0.86 & 1.19 & 0.5 \\
\hline 1.2 & 1.01 & 1.42 \\
\hline 1.59 & 1.28 & 1.93 \\
\hline
\end{tabular}

B

\begin{tabular}{|c|c|c|c|}
\hline TSS1500 & 0.95 & 1.2 & 0.67 \\
\hline TSS200 & 0.53 & 0.86 & 0.18 \\
\hline 5'UTR & 1.02 & 1.19 & 0.83 \\
\hline First Exon & 0.55 & 0.77 & 0.31 \\
\hline Gene Body & 1.14 & 0.98 & 1.32 \\
\hline 3'UTR & 1.42 & 0.86 & 2.03 \\
\hline ncRNA & 0.97 & 1.01 & 0.93 \\
\hline NA & 1.04 & 0.93 & 1.15 \\
\hline
\end{tabular}

C

\begin{tabular}{|c|c|c|c|c|c|}
\hline \multirow{2}{*}{ Enhancer } & \multirow{2}{*}{ H3K4me1 } & CD19+ & 0.99 & 1.45 & 0.53 \\
\hline & & CD3+ & 0.96 & 1.31 & 0.62 \\
\hline \multirow{2}{*}{ Enhancer } & \multirow{2}{*}{ H3К27ac } & CD19+ & 0.85 & 1.34 & 0.36 \\
\hline & & CD3+ & 0.95 & 1.27 & 0.63 \\
\hline \multirow{2}{*}{ Promoter } & \multirow{2}{*}{ H3K4me3 } & CD19+ & 0.63 & 1.11 & 0.14 \\
\hline & & CD3+ & 0.68 & 1.06 & 0.3 \\
\hline \multirow{2}{*}{$\begin{array}{l}\text { Transcribed } \\
\text { Gene Body }\end{array}$} & \multirow{2}{*}{ нзкз6mез } & CD19+ & 1.45 & 0.98 & 1.91 \\
\hline & & CD3+ & 1.31 & 0.95 & 1.66 \\
\hline \multirow{2}{*}{ Repressive } & \multirow{2}{*}{ H3K27me3 } & CD19+ & 0.4 & 0.6 & 0.2 \\
\hline & & CD3+ & 0.26 & 0.37 & 0.15 \\
\hline \multirow{2}{*}{\multicolumn{2}{|c|}{ Heterochromatin $\mathrm{H} 3 \mathrm{~K} 9 \mathrm{me} 3$}} & CD19+ & 0.64 & 0.29 & 0.99 \\
\hline & & CD3+ & 0.56 & 0.19 & 0.92 \\
\hline \multirow{2}{*}{ Open Chromatin } & \multirow{2}{*}{ DHS } & CD19+ & 0.74 & 1.25 & 0.23 \\
\hline & & CD3+ & 0.81 & 1.16 & 0.46 \\
\hline
\end{tabular}

progenitor cells independent of IFN activity. Coit $e t$ al $^{39}$ reported hypomethylation of IFN-induced genes in neutrophils from patients with SLE and speculate that the exposure to IFN during the disease course may induce methylation differences that will increase the responsiveness to IFN. Taken together, epigenetic changes in different cell types in pSS and SLE makes them poised for type I IFN expression, although the exact mechanisms remain to be elucidated. In concordance with others, we found that the hypomethylation of IFN-induced genes is largely driven by the antibody-positive patients. ${ }^{15}$ Our pathway analysis of DMCs also revealed IFN signalling among the top associated pathways. Monoclonal antibodies interfering with the IFN signalling pathway currently under development in SLE might be of interest in future clinical trials in selected patients with pSS.

Polymorphisms in genes of the type I IFN system have shown associations with pSS. ${ }^{9} 1040$ We therefore investigated whether genetic variants in known pSS associated loci mediate disease risk by influencing methylation levels at target $\mathrm{CpG}$ sites. We identified significant associations of genetic variants in DDX6-CXCR5, FAM167A-BLK, IL12A, IRF5-TNPO3, STAT4, TNIP1, and within the HLA region with DNA methylation, indicating that pSS GWAS risk alleles have the potential to affect DNA methylation levels.

For insights into functional mechanisms of DMCs in pSS we studied the regional genomic distribution of associated sites. We found distinct enrichment patterns for overlap with chromatin marks, where hypomethylated DMCs were enriched for enhancer marks and regions of open chromatin, while hypermethylated DMCs were highly under-represented in these functional regions. Hypomethylated $\mathrm{CpG}$ sites were also more prominent in promoter regions, indicating a putative transcriptional activation of genes with hypomethylation in these regions. 
Table 3 Top DMCs and the corresponding differential gene expression in CD19+ B cells in patients with primary Sjögren's syndrome (pSS) and healthy controls

\begin{tabular}{|c|c|c|c|c|c|c|c|c|c|c|c|}
\hline CpG site & $\begin{array}{l}\text { Gene } \\
\text { symbol }\end{array}$ & Gene name & Interferon-induced & $\begin{array}{l}\text { CpG island } \\
\text { information }\end{array}$ & $\begin{array}{l}\text { Gene } \\
\text { property }\end{array}$ & Enhancer* & Promotert & $\begin{array}{l}\text { Methylation } \\
\Delta \beta \neq\end{array}$ & $\begin{array}{l}\text { Methylation } \\
\text { p value§ }\end{array}$ & $\begin{array}{l}\text { Expression log2 } \\
\text { (FC) } \pi\end{array}$ & $\begin{array}{l}\text { Expression } \\
\text { p value** }\end{array}$ \\
\hline $\operatorname{cg} 21549285$ & $M X 1$ & MX dynamin-like GTPase 1 & Yes & Shore & 5'UTR & No & Yes & -0.34 & $2.3 \times 10^{-11}$ & 1.97 & $<5 \times 10^{-5}$ \\
\hline cg05696877 & IFI44L & Interferon-induced protein 44-like & Yes & Open sea & 5'UTR & No & No & -0.33 & $3.8 \times 10^{-20}$ & 3.79 & $<5 \times 10^{-5}$ \\
\hline cg03607951 & IFI44L & Interferon-induced protein 44-like & Yes & Open sea & TSS1500 & Yes & Yes & -0.30 & $2.6 \times 10^{-18}$ & 3.79 & $<5 \times 10^{-5}$ \\
\hline $\operatorname{cg} 23570810$ & IFITM1 & Interferon-induced transmembrane protein 1 & Yes & Shore & Gene body & Yes & No & -0.26 & $1.3 \times 10^{-10}$ & 1.63 & $<5 \times 10^{-5}$ \\
\hline cg05552874 & IFIT1 & $\begin{array}{l}\text { Interferon-induced protein with } \\
\text { tetratricopeptide repeats } 1\end{array}$ & Yes & Open sea & Gene body & Yes & Yes & -0.26 & $2.1 \times 10^{-16}$ & 4.44 & $<5 \times 10^{-5}$ \\
\hline cg06981309 & PLSCR1 & Phospholipid scramblase 1 & Yes & Shore & 5'UTR & No & Yes & -0.25 & $2.3 \times 10^{-12}$ & 1.64 & $<5 \times 10^{-5}$ \\
\hline $\operatorname{cg} 14293575$ & USP18 & Ubiquitin-specific peptidase 18 & Yes & Shelf & 5'UTR & No & No & -0.25 & $9.7 \times 10^{-14}$ & 3.27 & $<5 \times 10^{-5}$ \\
\hline cg03038262 & IFITM1 & Interferon-induced transmembrane protein 1 & Yes & Shore & $3^{\prime}$ UTR & Yes & No & -0.25 & $2.9 \times 10^{-10}$ & 1.63 & $<5 \times 10^{-5}$ \\
\hline cg22930808 & PARP9 & $\begin{array}{l}\text { Poly (ADP-ribose) polymerase family, } \\
\text { member } 9\end{array}$ & Yes & Shore & 5'UTR & Yes & No & -0.24 & $2.4 \times 10^{-10}$ & 1.89 & $<5 \times 10^{-5}$ \\
\hline $\operatorname{cg} 00959259$ & PARPG & $\begin{array}{l}\text { Poly (ADP-ribose) polymerase family, } \\
\text { member } 9\end{array}$ & Yes & Shore & 5'UTR & Yes & No & -0.24 & $1.3 \times 10^{-13}$ & 1.89 & $<5 \times 10^{-5}$ \\
\hline $\operatorname{cg} 22862003$ & MX1 & MX dynamin-like GTPase 1 & Yes & Shore & TSS1500 & Yes & Yes & -0.22 & $5.8 \times 10^{-12}$ & 1.97 & $<5 \times 10^{-5}$ \\
\hline cg10549986 & RSAD2 & $\begin{array}{l}\text { Radical S-adenosyl methionine domain } \\
\text { containing } 2\end{array}$ & Yes & Open sea & First exon & Yes & Yes & -0.21 & $1.4 \times 10^{-17}$ & 3.19 & $<5 \times 10^{-5}$ \\
\hline cg16810031 & ZPBP2 & Zona pellucida binding protein 2 & No & CpG island & TSS1500 & Yes & No & 0.21 & $7.4 \times 10^{-8}$ & n.a. & n.a. \\
\hline $\operatorname{cg} 25330422$ & STAT3 & $\begin{array}{l}\text { Signal transducer and activator of } \\
\text { transcription } 3\end{array}$ & Yes & Shelf & 3'UTR & Yes & Yes & 0.22 & $3.2 \times 10^{-13}$ & -0.09 & n.s. \\
\hline
\end{tabular}

*Genomic location of differentially methylated CpG site (DMC) overlapping H3K4me1 (poised enhancer mark) and/or H3K27ac (active enhancer mark) peak in reference CD19+ B cells, tGenomic location of DMC overlapping H3K4me3 (active promoter mark) peak in reference CD19+ B cells.

¥Methylation $\Delta \beta$ refers to the difference in mean methylation $\beta$ ( $\beta$-values) between patients with pSS and healthy controls. A negative $\Delta \beta$ represents hypomethylation in the patients.

$\S p$ Value of the association between CpG methylation and pSS (significance threshold $p<1.3 \times 10^{-7}$; Bonferroni corrected).

TDifferential gene expression as $\log _{2}(\mathrm{FC})$ between patients with $\mathrm{pSS}$ and healthy controls.

FC, fold change; n.a., not available; n.s., not significant; TSS, transcription start site. 
Table 4 Methylation quantitative trait loci (metQTL) of genetic variants associated with primary Sjögren's syndrome (pSS) at genome-wide significance and the DNA methylation association of the most significant metQTL CPG site

\begin{tabular}{|c|c|c|c|c|}
\hline Gene locus & $\begin{array}{l}\text { pSS associated } \\
\text { SNP* }\end{array}$ & $\begin{array}{l}\text { Top CpG } \\
\text { site }\end{array}$ & $\begin{array}{l}\text { metQTL } \\
\text { p Value }\end{array}$ & $\begin{array}{l}\text { pSS } \\
\text { EWAS } \\
\text { p Value }\end{array}$ \\
\hline$D D X 6-C X C R 5$ & rs4936443 & cg09144398 & $2.5 \times 10^{-3}$ & $9.9 \times 10^{-4}$ \\
\hline FAM167A-BLK & rs2736345 & $\operatorname{cg} 21775007$ & $2.1 \times 10^{-9}$ & 0.02 \\
\hline IL12A & rs485497 & $\operatorname{cg} 25829945$ & 0.02 & 0.03 \\
\hline IL12A & rs583911 & cg07906551 & 0.01 & $4.5 \times 10^{-8}$ \\
\hline IRF5-TNPO3 & rs4728142 & cg04864179 & $4.8 \times 10^{-9}$ & $4.8 \times 10^{-13}$ \\
\hline IRF5-TNPO3 & rs17339836 & $\operatorname{cg} 04864179$ & $4.6 \times 10^{-4}$ & $4.8 \times 10^{-13}$ \\
\hline IRF5-TNPO3 & rs10954213 & cg04864179 & $6.9 \times 10^{-6}$ & $4.8 \times 10^{-13}$ \\
\hline STAT4 & rs13426947 & cg15325732 & 0.01 & 0.03 \\
\hline TNIP1 & rs6579837 & cg19084508 & 0.03 & $2.5 \times 10^{-3}$ \\
\hline TNIP1 & rs7732451 & cg15989436 & 0.04 & $1.5 \times 10^{-13}$ \\
\hline HLA-DRA & rs3135394 & cg25645491 & $8.2 \times 10^{-13}$ & $2.0 \times 10^{-3}$ \\
\hline$H L A-D Q B 1$ & rs115575857 & cg07180897 & $3.0 \times 10^{-70}$ & $8.9 \times 10^{-7}$ \\
\hline$H L A-D Q B 1$ & rs3129716 & cg07180897 & $3.0 \times 10^{-70}$ & $8.9 \times 10^{-7}$ \\
\hline$H L A-D Q A 1$ & rs9271588 & cg08269402 & $4.1 \times 10^{-51}$ & $5.0 \times 10^{-4}$ \\
\hline
\end{tabular}

* Single nucleotide polymorphism (SNP) associated with pSS at genome-wide significance reported by Lessard et al. ${ }^{9}$

EWAS, epigenome-wide association study.

Interestingly, distinct hypomethylation and increased gene expression of the PARP9 (BAL1) gene were found in CD19+ $\mathrm{B}$ cells. Whereas BAL1 has been shown to be overexpressed in diffuse large B cell lymphomas, ${ }^{41}$ none of our patients sampled for CD19+ B cells had a previous lymphoma. Disease annotation analysis of the top DMCs in whole blood also pointed to an extensive enrichment of genes associated with lymphoproliferative disorders, including B cell non-Hodgkin's lymphoma. This is intriguing since pSS is the autoimmune disease which displays the highest risk for lymphoma development. ${ }^{42}$ Whether aberrant methylation in target genes contributes to lymphoma development is an important topic for future studies.

The strongest genetic association for pSS is found in the HLA region. ${ }^{9}$ Although none of the most significantly associated DMCs were annotated to HLA genes, we nonetheless identified a considerable number of significant signals within this region with the majority being hypomethylated in patients with pSS, possibly pointing to an increased expression of alternatively spliced antigenic transcripts in pSS. ${ }^{43} 44$ Analysing X chromosomal DNA methylation we observed several DMCs in genes with central roles in connecting innate and adaptive immunity, such as TLR8 and CD4OL, as well as in miRNAs. ${ }^{45}$ Altered miRNA expression constitutes another epigenetic mechanism implicated in pSS pathogenesis, ${ }^{46}$ and the potential role of miRNA-223 hypomethylation in pSS pathogenesis warrants further investigation.

In our study we used a well known reference based method for cell type estimation of whole blood samples. ${ }^{23}{ }^{24}$ In the purified CD19+ B cells compared with the whole blood analysis, we noted a larger mean difference in methylation levels between cases and controls for many associated $\mathrm{CpG}$ sites, including at MX1 and IFI44L, perhaps indicating the advantage of a single cell type in the analysis. However, the smaller number of individuals from which purified cells could be obtained, compared with DNA extracted from whole blood, still meant that fewer DMCs were detected studying B cells. Due to the low absolute number of B cells in serum from patients with pSS we were not able to assess methylation patterns in different B cell subtypes. Minor salivary gland biopsies consist of different cell types including epithelial and acinar cells in both patients with pSS and controls, but also inflammatory cells in the patients' biopsies. Therefore we cannot deduce the cell types that are responsible for the difference in methylation between diseased and normal glands, and the results must be interpreted with caution. Nevertheless, it is noteworthy that the strongest association was found in OAS2, an IFN-induced gene involved in the innate immune response. ${ }^{31}$ The minor salivary glands are known targets for IFN where both increased IFN- $\alpha$ levels and an IFN signature have been demonstrated. ${ }^{3-5}$

In conclusion, our study of epigenetic profiles in multiple tissues in pSS using a large collection of patients and controls has replicated the previously reported hypomethylation of IFN-regulated genes in pSS and identified numerous new associations. We report hypomethylation in regulatory enhancer and promotor regions and show for the first time that hypomethylation of IFN-regulated genes in B cells corresponds to an increase in gene expression. Evidence for genetic control of methylation levels at known pSS risk loci is presented. Independent replication in cells from patients with pSS and controls will be required to confirm these novel findings. Studying the epigenetic basis of pSS will hopefully increase our understanding of the disease mechanisms and guide the search for novel and more specific therapeutic targets.

Acknowledgements The authors thank Rezvan Kiani and Karolina Tandre for collecting samples from patients and controls. Genotyping, epigenotyping and RNA-sequencing were performed at the SNP\&SEQ Technology Platform at the National Genomics Infrastructure (NGI) hosted by Science for Life Laboratory in Uppsala, Sweden (http://www.genotyping.se; http://www.sequencing.se). The authors thank Andrei Alexsson, Tomas Axelsson, Anna Haukkala, Maria Hägglund Charlotta Jakobsson and Anders Lundmark for excellent technical assistance. The authors especially thank all patients and blood donors who contributed samples to this study.

Contributors JI-K, JKS, KBN, RO, A-CS and GN designed the study; LS, LR, M-LE and GN collected patient and control material and clinical data; JI-K and JKS performed the experiments; JI-K, JKS, JCA and JN analysed the data; JI-K, JKS and GN drafted the manuscript and all authors read and accepted the final version of the manuscript. JI-K and JKS contributed equally to the study.

Funding This study was supported by grants from the Knut and Alice Wallenberg Foundation, the Swedish Research Council for Medicine and Health (Dnr 521-2014-2263 ACS and Dnr 521-2013-2830 LR), the Gustav V: 80-year Foundation, Combine, the Swedish Society of Medicine and the Swedish

Rheumatism Association. JKS was supported by a Swedish Research Council postdoc grant (Dnr 350-2012-256). The SNP\&SEQ Technology Platform is supported by the Swedish Research Council (VR-RFI), Science for Life Laboratory and the Knut and Alice Wallenberg Foundation.

Competing interests None declared.

\section{Patient consent Obtained.}

Ethics approval The study was approved by the Regional Ethics board in Uppsala No. 97358, 217/2006 and 013/2009.

Provenance and peer review Not commissioned; externally peer reviewed.

Data sharing statement Normalised or raw intensity data of the HM450K BeadChips are available upon request from the authors on a collaborative basis.

Open Access This is an Open Access article distributed in accordance with the Creative Commons Attribution Non Commercial (CC BY-NC 4.0) license, which permits others to distribute, remix, adapt, build upon this work non-commercially, and license their derivative works on different terms, provided the original work is properly cited and the use is non-commercial. See: http://creativecommons.org/ licenses/by-nc/4.0/

\section{REFERENCES}

1 Jonsson R, Bolstad Al, Brokstad KA, et al. Sjogren's syndrome-a plethora of clinical and immunological phenotypes with a complex genetic background. Ann N Y Acad Sci 2007;1108:433-47. 
2 Nocturne $G$, Mariette $X$. Advances in understanding the pathogenesis of primary Sjögren's syndrome. Nat Rev Rheumatol 2013;9:544-56.

3 Båve U, Nordmark G, Lövgren T, et al. Activation of the type I interferon system in primary Sjögren's syndrome: a possible etiopathogenic mechanism. Arthritis Rheum 2005;52:1185-95.

4 Gottenberg JE, Cagnard N, Lucchesi C, et al. Activation of IFN pathways and plasmacytoid dendritic cell recruitment in target organs of primary Sjögren's syndrome. Proc Natl Acad Sci USA 2006;103:2770-5.

5 Hjelmervik TO, Petersen $\mathrm{K}$, Jonassen I, et al. Gene expression profiling of minor salivary glands clearly distinguishes primary Sjögren's syndrome patients from healthy control subjects. Arthritis Rheum 2005;52:1534-44.

6 Emamian ES, Leon JM, Lessard CJ, et al. Peripheral blood gene expression profiling in Sjögren's syndrome. Genes Immun 2009;10:285-96.

7 Brkic Z, Maria NI, van Helden-Meeuwsen CG, et al. Prevalence of interferon type I signature in CD14 monocytes of patients with Sjögren's syndrome and association with disease activity and BAFF gene expression. Ann Rheum Dis 2013;72:728-35.

8 Theander $\mathrm{E}$, Henriksson $\mathrm{G}$, Ljungberg $\mathrm{O}$, et al. Lymphoma and other malignancies in primary Sjögren's syndrome: a cohort study on cancer incidence and lymphoma predictors. Ann Rheum Dis 2006:65:796-803.

9 Lessard CJ, Li H, Adrianto I, et al. Variants at multiple loci implicated in both innate and adaptive immune responses are associated with Sjögren's syndrome. Nat Genet 2013:45:1284-92.

10 Li Y, Zhang K, Chen $\mathrm{H}$, et al. A genome-wide association study in Han Chinese identifies a susceptibility locus for primary Sjögren's syndrome at 7q11.23. Nat Genet 2013;45:1361-5.

11 Ice JA, Li H, Adrianto I, et al. Genetics of Sjögren's syndrome in the genome-wide association era. J Autoimmun 2012;39:57-63.

12 Konsta OD, Thabet $Y$, Le Dantec $C$, et al. The contribution of epigenetics in Sjögren's Syndrome. Front Genet 2014;5:71.

13 Bibikova M, Barnes B, Tsan C, et al. High density DNA methylation array with single CpG site resolution. Genomics 2011;98:288-95.

14 Altorok N, Coit $\mathrm{P}$, Hughes $\mathrm{T}$, et al. Genome-wide DNA methylation patterns in naive CD4+ T cells from patients with primary Sjögren's syndrome. Genomics 2014;66:731-9.

15 Miceli-Richard C, Wang-Renault SF, Boudaoud S, et al. Overlap between differentially methylated DNA regions in blood $B$ lymphocytes and genetic at-risk loci in primary Sjogren's syndrome. Ann Rheum Dis 2016;75:933-40.

16 Bernstein BE, Stamatoyannopoulos JA, Costello JF, et al. The NIH Roadmap Epigenomics Mapping Consortium. Nat Biotechnol 2010;28:1045-8.

17 Vitali C, Bombardieri S, Jonsson R, et al. Classification criteria for Sjogren's syndrome: a revised version of the European criteria proposed by the American-European Consensus Group. Ann Rheum Dis 2002;61:554-8.

18 Nordmark G, Wang C, Vasaitis L, et al. Association of genes in the NF-kB pathway with antibody-positive primary Sjogren's syndrome. Scand I Immunol 2013;78:447-54.

19 Berggren O, Alexsson A, Morris DL, et al. IFN-alpha production by plasmacytoid dendritic cell associations with polymorphisms in gene loci related to autoimmune and inflammatory diseases. Hum Mol Genet 2015;24:3571-81.

20 R Core Development Team. R: A Language and Environment for Statistical Computing. Vienna, Austria: R Foundation for Statistical Computing, 2011

21 Aryee MJ, Jaffe AE, Corrada-Bravo H, et al. Minfi: a flexible and comprehensive Bioconductor package for the analysis of Infinium DNA methylation microarrays. Bioinformatics 2014;30:1363-9.

22 Maksimovic J, Gordon L, Oshlack A. SWAN: Subset-quantile within array normalization for illumina infinium HumanMethylation450 BeadChips. Genome Biol 2012;13:R44.

23 Houseman EA, Accomando WP, Koestler DC, et al. DNA methylation arrays as surrogate measures of cell mixture distribution. BMC bioinformatics 2012;13:86.
24 Reinius LE, Acevedo N, Joerink M, et al. Differential DNA methylation in purified human blood cells: implications for cell lineage and studies on disease susceptibility. PLOS ONE 2012;7:e41361.

25 DeLuca DS, Levin IZ, Sivachenko A, et al. RNA-SeQC: RNA-seq metrics for quality control and process optimization. Bioinformatics 2012;28:1530-2.

26 Trapnell C, Roberts A, Goff L, et al. Differential gene and transcript expression analysis of RNA-seq experiments with TopHat and Cufflinks. Nat Protoc 2012;7:562-78.

27 Kim D, Pertea G, Trapnell C, et al. TopHat2: accurate alignment of transcriptomes in the presence of insertions, deletions and gene fusions. Genome Biol 2013;14:R36.

28 Purcell S, Neale B, Todd-Brown K, et al. PLINK: a tool set for whole-genome association and population-based linkage analyses. Am J Hum Genet 2007:81:559-75

29 Haller O, Kochs G. Human MxA protein: an interferon-induced dynamin-like GTPase with broad antiviral activity. J Interferon Cytokine Res 2011;31:79-87.

30 Wang SS, Betz AG, Reed RR. Cloning of a novel Olf-1/EBF-like gene, O/E-4, by degenerate oligo-based direct selection. Mol Cell Neurosci 2002;20:404-14.

31 Hovanessian AG, Justesen J. The human 2'-5'oligoadenylate synthetase family: unique interferon-inducible enzymes catalyzing $2^{\prime}-5^{\prime}$ instead of $3^{\prime}-5^{\prime}$ phosphodiester bond formation. Biochimie 2007:89:779-88.

32 Nordmark G, Eloranta ML, Ronnblom L. Primary Sjogren's syndrome and the type I interferon system. Curr Pharm Biotechnol 2012;13:2054-62.

33 Nezos A, Gravani F, Tassidou A, et al. Type I and II interferon signatures in Sjogren's syndrome pathogenesis: contributions in distinct clinical phenotypes and Sjogren's related lymphomagenesis. J Autoimmun 2015;63:47-58.

34 Rönnblom L, Alm GV, Eloranta ML. The type I interferon system in the development of lupus. Semin Immunol 2011;23:113-21.

35 Maria NI, Brkic Z, Waris M, et al. MxA as a clinically applicable biomarker for identifying systemic interferon type I in primary Sjogren's syndrome. Ann Rheum Dis 2014;73:1052-9

36 Coit $\mathrm{P}$, Jeffries $\mathrm{M}$, Altorok N, et al. Genome-wide DNA methylation study suggests epigenetic accessibility and transcriptional poising of interferon-regulated genes in naive CD4+ T cells from lupus patients. J Autoimmun 2013;43:78-84.

37 Absher DM, Li X, Waite LL, et al. Genome-wide DNA methylation analysis of systemic lupus erythematosus reveals persistent hypomethylation of interferon genes and compositional changes to CD4+ T-cell populations. PLoS Genet 2013;9:e1003678.

38 Zhao M, Liu S, Luo $S$, et al. DNA methylation and mRNA and microRNA expression of SLE CD4+ T cells correlate with disease phenotype. J Autoimmun 2014;54:127-36.

39 Coit $\mathrm{P}$, Yalavarthi $\mathrm{S}$, Ognenovski $\mathrm{M}$, et al. Epigenome profiling reveals significant DNA demethylation of interferon signature genes in lupus neutrophils. J Autoimmun 2015:58:59-66.

40 Nordmark G, Kristjansdottir G, Theander E, et al. Association of EBF1, FAM167A (C8orf13)-BLK and TNFSF4 gene variants with primary Sjögren's syndrome. Genes Immun 2011:12:100-9.

41 Camicia R, Bachmann SB, Winkler HC, et al. BAL1/ARTD9 represses the anti-proliferative and pro-apoptotic IFNg-STAT1-IRF1-p53 axis in diffuse large B-cell lymphoma. J Cell Sci 2013;126(Pt 9):1969-80.

42 Baecklund E, Smedby KE, Sutton LA, et al. Lymphoma development in patients with autoimmune and inflammatory disorders—what are the driving forces? Semin Cancer Biol 2014;24:61-70.

43 Lev Maor G, Yearim A, Ast G. The alternative role of DNA methylation in splicing regulation. Trends Genet 2015;31:274-80.

44 Graves M, Benton M, Lea R, et al. Methylation differences at the HLA-DRB1 locus in CD4+ T-Cells are associated with multiple sclerosis. Mult Scler 2013;20:1033-41.

45 Haneklaus M, Gerlic M, O'Neill LA, et al. miR-223: infection, inflammation and cancer. J Intern Med 2013;274:215-26.

46 Alevizos I, Alexander S, Turner RJ, et al. MicroRNA expression profiles as biomarkers of minor salivary gland inflammation and dysfunction in Sjögren's syndrome. Arthritis Rheum 2011;63:535-44. 